Pacific Journal of Mathematics

ON THE SEMISIMPLICITY OF GROUP RINGS OF LINEAR 


\title{
ON THE SEMISIMPLICITY OF GROUP RINGS OF LINEAR GROUPS II
}

\author{
D. S. PAssman
}

In this paper we continue the study of the semisimplicity problem for group rings of linear groups. We consider the case in which the characteristics of the two fields involved are both equal to $p>0$ and we obtain appropriate necessary and sufficient conditions in terms of the abstract structure of the group.

Let $K[G]$ denote the group ring of $G$ over the field $K$. In this paper we study the semisimplicity problem for $K[G]$ with $G$ a linear group. If char $K=0$ and if $G$ is a linear group over any field, then it is trivial to see that $J K[G]=0$. Thus the only case of interest occurs when char $K=p>0$. A study of this situation was initiated by A. E. Zalesskii in [4] and continued somewhat in [3]. Here we solve the problem in case $G$ is a linear group over a field $L$ and char $L=$ char $K$. Before we can properly state the result it is necessary to describe a certain characteristic subgroup $\mathscr{L}(G)$ of $G$. Therefore, we postpone the statement until the next section. We follow the notation of [2] and [3].

1. Normal $p$-subgroups. Let $G$ be a linear group over a field $L$ of characteristic $p>0$. That is, $G$ is a subgroup of the group of units of $L_{u}$, the ring of $u \times u$ matrices over $L$. Of course $G$ is also contained in $\widetilde{L}_{u}$, where $\widetilde{L}$ is the algebraic closure of $L$ and thus without loss of generality we may assume that $L$ is algebraically closed. Thus for the remainder of this work $L$ will denote a fixed algebraically closed field of $\operatorname{char} p>0$ and any subgroup of $L_{u}$ for any $u$ will be called an $L$-linear group.

It is apparent from [4] that a necessary ingredient here must be a consideration of the normal $p$-subgroups of $G$. We start with a few elementary observations. If $G$ is any group let $\boldsymbol{O}_{p}(G)$ denote its maximal normal $p$-subgroup. It is clear that $\boldsymbol{O}_{p}(G)$ always exists. If $G \leqq L_{u}$ we let $L G$ donote its $L$-linear span. Thus certainly $L G$ is an $L$-subalgebra of $L_{u}$.

Lemma 1.1. Let $G$ be an L-linear group. Then

(i) $\boldsymbol{O}_{p}(G)$ is a nilpotent group.

(ii) $G / O_{p}(G)$ is an L-linear group.

(iii) If $\boldsymbol{O}_{p}(G)=\langle 1\rangle$, then $G$ can be represented as an $L$-linear group in such a way that $L G$ is semisimple. 
(iv) If $L G$ is semisimple and $H \triangleleft G$ then $L H$ is semisimple.

Proof. Observe that $L G$ is a finite dimensional $L$-algebra so $J L G$, its Jacobson radical, is nilpotent. We start by proving (iv). If $x \in G$ then since $H \triangleleft G, x$ normalizes $H$ and hence clearly $x$ acts as an algebra automorphism on $L H$. Since $J L H$ is characteristic in $L H$ we have $x^{-1}(J L H) x=J L H$ so $(J L H) x=x(J L H)$. Thus since $L G$ is spanned by all such $x$ we obtain easily $(J L H)(L G)=(L G)(J L H)$. Now $J L H$ is nilpotent and therefore by the above so is the ideal $(J L H)(L G)$. Thus $(J L H)(L G) \leqq J L G=0$ and $J L H=0$. This yields (iv).

Now let $\mu: L G \rightarrow L G / J L G$ be the natural map and let $P=\{g \in G \mid \mu(g)=$ 1\}. Since $G \subseteq L G, P$ is a subgroup of $U=\{1+\alpha \mid \alpha \in J L G\} \cong L G$. Now $J L G$ is nilpotent and char $L=p>0$ so we see easily that $U$ is a nilpotent $p$-group. Thus $P$ is a nilpotent $p$-group and $P \leqq \boldsymbol{O}_{p}(G)$.

Now $\mu(L G)=L G / J L G$ is a finite dimensional $L$-algebra so it is contained in $L_{w}$ for some integer $w$. Furthermore, $L G / J L G$ contains the group $\bar{G}=G / P$ and is clearly spanned by it. This shows that $\bar{G}$ is an $L$-linear group with $L \bar{G}$ semisimple. If $\boldsymbol{O}_{p}(G)=\langle 1\rangle$ then certainly $P=\langle 1\rangle$ so $G=\bar{G}$ and (iii) is proved.

Observe that if we show that $P=\boldsymbol{O}_{p}(G)$ then (i) and (ii) will follow and to do this we need only show that $\bar{Q}=\boldsymbol{O}_{p}(\bar{G})=\langle 1\rangle$. Since $L \bar{G}$ is semisimple, part (iv) and $\bar{Q} \triangleleft \bar{G}$ implies that $L \bar{Q}$ is also semisimple. Let $I$ be the subalgebra of $L \bar{Q}$ spanned by all $1-\imath$ with $x \in \bar{Q}$. Then $I$ is an ideal of $L \bar{Q}$ and $I$ is a finite dimensional algebra (without 1) spanned by the nilpotent elements $1-x$. As is well known (see for example the proof of Lemma 10.1 (ii) of [2]) this implies that $I$ is nilpotent so $I \subseteq J L \bar{Q}=0$. If $x \in \bar{Q}$ then $1-x \in I=0$ so $x=1$. Thus $\bar{Q}=\langle 1\rangle$ and the lemma is proved.

Let $G$ be any group and let $H$ be a subgroup of $G$. We set

$$
\boldsymbol{D}_{G}(H)=\left\{x \in G \mid\left[H: \boldsymbol{C}_{H}(x)\right]<\infty\right\} \text {. }
$$

Clearly $\boldsymbol{D}_{G}(H)$ is a subgroup of $G$ and if $H$ is normal or characteristic in $G$ then so is $D_{G}(H)$. Furthermore,

$$
\boldsymbol{D}_{G}(G)=\Delta(G)=\left\{x \in G \mid\left[G: \boldsymbol{C}_{G}(x)\right]<\infty\right\}
$$

is the $F$. C. subgroup of $G$. Finally $\Delta^{p}(G)$ is defined to be the subgroup of $\Delta(G)$ generated by all $p$-elements, that is elements whose order is a power of $p$. We say that $G$ is a $\Delta$-group if $G=\Delta(G)$.

Lemma 1.2. Let $G$ be an L-linear group.

(i) If $H \triangleleft G$ and $G=D_{G}(H)$ then $[H: H \cap Z(G)]<\infty$.

(ii) If $\boldsymbol{O}_{p}(G)=\langle 1\rangle$ then $\Delta^{p}(G)$ is finite. 
Proof. Since $L G$ is finite dimensional we can choose some finite number of group elements $x_{1}, x_{2}, \cdots, x_{n}$ which span $L G$. By assumption for each $i,\left[H: C_{H}\left(x_{i}\right)\right]<\infty$ and thus by Lemma 1.1 of [2], $[H: Z]<\infty$ where $Z=\bigcap_{1}^{n} C_{H}\left(x_{i}\right)$. Now $Z \subseteq L G$ is centralized by a spanning set so it is, therefore, centralized by all of $L G$ and hence by all of $G$. This shows that $Z \cong Z(G)$ and thus (i) follows.

Suppose $O_{p}(G)=\langle 1\rangle$ and set $H=\Delta^{p}(G)$. Then $H=\Delta(H)$ so by part (i) applied to $H$ we conclude that $[H: Z(H)]<\infty$. Now $\boldsymbol{O}_{p}(G)=\langle 1\rangle$ and $Z(H) \triangleleft G$ so $O_{p}(Z(H))=\langle 1\rangle$ and since $Z(H)$ is abelian this says that $Z(H)$ has no elements of order $p$. Thus $\Delta^{p}(Z(H))=\langle 1\rangle$. On the other hand, since [ $\left.\Delta^{p}(G): Z(H)\right]<\infty$, Lemma 19.3 (v) of [2] implies that $\left[\boldsymbol{Z}(H): \Delta^{p}(\boldsymbol{Z}(H))\right]<\infty$. Thus $\Delta^{p}(\boldsymbol{Z}(H))=\langle 1\rangle$ yields $|\boldsymbol{Z}(H)|<\infty$ and hence $|H|<\infty$. This completes the proof.

Let $G$ be any group. We define a characteristic subgroup $\mathscr{L}(G)$ of $G$ as follows. Let $P=\boldsymbol{O}_{p}(G)$ and set $G^{*}=D_{G}(P)$ so that $G^{*} \cap P=$ $\boldsymbol{D}_{P}(P)=\Delta(P)$. Then $\mathscr{L}(G)$ is the subgroup of $G^{*}$ given by

$$
G^{*} \supseteqq \mathscr{L}(G) \supseteqq \Delta(P), \quad \mathscr{L}(G) / \Delta(P)=\Delta^{p}\left(G^{*} / \Delta(P)\right) \text {. }
$$

LemmA 1.3. Let $G$ be an L-linear group. Then with the above notation $[\mathscr{L}(G): \Delta(P)]$ is finite and $\mathscr{L}(G)$ is a characteristic 4 -subgroup of $G$.

Proof. $\mathscr{L}(G)$ is clearly characteristic by its construction. Now $G^{*} \triangleleft G$ so $\boldsymbol{O}_{p}\left(G^{*}\right) \subseteq \boldsymbol{O}_{p}(G)=P$ and thus $\boldsymbol{O}_{p}\left(G^{*}\right)=\Delta(P)$. Therefore, by Lemma 1.1 (ii), $G^{*} / \Delta(P)$ is an $L$-linear group and certainly $\boldsymbol{O}_{p}\left(G^{*} / \Delta(P)\right)=\langle 1\rangle$. Thus Lemma 1.2 (ii) implies that $\Delta^{p}\left(G^{*} / \Delta(P)\right)$ is finite and we see that $[\mathscr{L}(G): \Delta(P)]$ is finite. Furthermore, since clearly $G^{*}=\boldsymbol{D}_{G^{*}}(\Delta(P))$, Lemma 1.2 (ii) yields $\left[\Delta(P): \Delta(P) \cap \boldsymbol{Z}\left(G^{*}\right)\right]<\infty$ and this and the above show that $\mathscr{L}(G)$ has a center of finite index. Therefore, $\mathscr{L}(G)$ is a $\Delta$-group.

We can now state our main result. If $H$ is a subgroup of $G$ we say that $H$ has locally finite index in $G$ and write $[G: H]=1$.f. if for all finitely generated subgroups $S$ of $G$ we have $[S: S \cap H]<\infty$.

THEOREM. Let $K$ be a field of characteristic $p>0$ and let $G$ be a linear group over a field of the same characteristic $p$. Then $J K[G] \neq 0$ if and only if there exists an element $h \in \mathscr{L}(G)$ of order $p$ with $\left[G: C_{G}(h)\right]=l . f$.

Observe that the above necessary and sufficient conditions concern the abstract structure of $G$ and not how $G$ is written as a linear group. 
2. The case: $\boldsymbol{O}_{p}(G)=\langle 1\rangle$. The linear groups with $\boldsymbol{O}_{p}(G)=\langle 1\rangle$ were studied in [4] under the additional assumption that $K=L$, that is the two fields are the same, and the semisimplicity problem was solved in that case. Here we modify the original argument slightly to handle the case in which $K$ and $L$ are different.

If $S$ is a subset of any group $G$ we say that $S$ has finite index in $G$ and write $[G: S]<\infty$ if $G$ can be written as a finite union, $G=\bigcup_{1}^{n} S x_{i}$, of right translates of $S$.

Lemma 2.1. Let $G$ be an L-linear group and let $T_{1}, T_{2}, \cdots, T_{j}$ be a finite number of $L$-subspaces of $L G$ properly smaller than $L G$. Let $S$ be a subset of $G$ and suppose that

$$
G=S \cup \bigcup_{1}^{j}\left(G \cap T_{i}\right)
$$

Then either $[G: S]<\infty$ or $G=\bigcup_{1}^{j}\left(G \cap T_{i}\right)$ and $G$ has a subgroup $H$ of finite index with $L H \neq L G$.

Proof. We assume that $[G: S]$ is infinite and we consider all ways of writing $G$ as a finite union

$$
G=\bigcup_{1}^{s} S x_{i} \cup \bigcup_{1}^{t}\left(G \cap M_{i}\right)
$$

where $x_{i} \in G$ and the $M_{i}$ are $L$-subspaces of $L G$ each contained in some $T_{i^{\prime}}$. By assumption such a decomposition exists. For each such union we associate an ordered pair $(d, r)$ where $d=\max \operatorname{dim} M_{i}$ and $r$ is the number of $M_{i}$ of dimension $d$. We say $\left(d_{1}, r_{1}\right)<\left(d_{2}, r_{2}\right)$ if $d_{1}<d_{2}$ or $d_{1}=d_{2}$ and $r_{1}<r_{2}$. This then is a well ordering and assume the above union is so chosen that $(d, r)$ is minimal. By definition $d<\operatorname{dim} L G$. We may assume that $\operatorname{dim} M_{i}=d$ for $i=1,2, \cdots, r$. Note that the $M_{i}$ terms must occur since $[G: S]=\infty$.

Fix $k \leqq r$ and $g \in G$. Then

$$
\left(G \cap M_{k}\right) g \leqq G=\bigcup_{1}^{s} S x_{i} \cup \bigcup_{1}^{t}\left(G \cap M_{i}\right)
$$

so

$$
\begin{aligned}
G \cap M_{k} & \subseteq \bigcup_{1}^{s} S x_{i} g^{-1} \cup \bigcup_{1}^{t}\left(G \cap M_{i}\right) g^{-1} \\
& =\bigcup_{1}^{s} S x_{i} g^{-1} \cup \bigcup_{1}^{t}\left(G \cap M_{i} g^{-1}\right)
\end{aligned}
$$

and thus

$$
G \cap M_{k} \leqq \bigcup_{1}^{s} S x_{i} g^{-1} \cup \bigcup_{1}^{t}\left(G \cap\left(M_{i} g^{-1} \cap M_{k}\right)\right)
$$


Thus replacing the term $G \cap M_{k}$ in the original union by the above yields a new such union with the subspace $M_{k}$ replaced by the finitely many subspaces $M_{i} g^{-1} \cap M_{k}$ for $i=1,2, \cdots, t$. If $\operatorname{dim}\left(M_{i} g^{-1} \cap M_{k}\right)<$ $\operatorname{dim} M_{k}$ for all $i$, we then get a new decomposition with some smaller parameter $\left(d^{\prime}, r^{\prime}\right)$. Since this cannot happen we conclude that for some $i, M_{i} g^{-1} \cap M_{k}=M_{k}$ or $M_{i} \supseteqq M_{k} g$. Since $M_{k}$ has the largest dimension of all the subspaces we therefore have $M_{i}=M_{k} g$ for some $i \leqq r$.

We have therefore shown that $G$ permutes by right multiplication the subspaces $M_{1}, M_{2}, \cdots, M_{r}$ and hence if $H$ is the stabilizer of $M_{1}$ then $[G: H]<\infty$. If $L H=L G$ then $M_{1} H=M_{1}$ implies that $M_{1}(L H)=M_{1}$ and then $M_{1} G=M_{1}$. Again by the minimality of $(d, r)$ and $[G: S]=\infty$ we have $G \cap M_{1} \neq \varnothing$ so let $y \in G \cap M_{1}$. Then $M_{1} G=M_{1}$ yields $M_{1} \supseteqq y G=G$. Thus $M_{1} \supseteqq L G$, a contradiction. This shows that $L H \neq L G$ and therefore $L H$ is a proper subalgebra of $L G$.

Finally let $1=g_{1}, g_{2}, \cdots, g_{m}$ be a set of right coset representatives for $H$ in $G$. By renumbering the $M_{i}$ 's if necessary we may assume that $M_{1} g_{i}=M_{i}$. Let $T_{i^{\prime}}$ be chosen with $M_{i} \subseteq T_{i^{\prime}}$. Now $M_{1} H=M_{1}$ yields $y H \subseteq M_{1}$ so $y H g_{i} \subseteq M_{1} g_{i}=M_{i}$. Thus

$$
G=y G=\bigcup_{1}^{m} y H g_{i} \leqq \bigcup_{1}^{m} M_{i} \subseteq \bigcup_{1}^{m} T_{i^{\prime}}
$$

so clearly $G=\bigcup_{1}^{j}\left(G \cap T_{i}\right)$ and the lemma is proved.

For the remainder of this work we let $K$ denote a fixed field of characteristic $p$. If $G$ is a group and if $x, y \in G$ we use $x \sim{ }_{G} y$ to indicate that $x$ and $y$ are conjugate in $G$.

LEMMA 2.2. Let $\alpha=\sum_{i=1}^{k} a_{i} g_{i} \in K[G], \alpha \neq 0$ and suppose that $\alpha$ is nilpotent. Then for some $i \neq j$ and some integer $n$ we have $\boldsymbol{g}_{i}^{p^{n}} \sim{ }_{G} \boldsymbol{g}_{j}^{p^{n}}$.

Proof. Let $S$ denote the subspace of $K[G]$ spanned by all Lie products $[\beta, \gamma]=\beta \gamma-\gamma \beta$ with $\beta, \gamma \in K[G]$. Then $S$ is spanned by all Lie products $[x, y]=x y-y x$ with $x, y \in G$. Now $y x=x^{-1}(x y) x$ so $y x \sim{ }_{G} x y$ and, therefore, we see that if $\delta \in S$ then the sum of the coefficients of $\delta$ over any conjugacy class of $G$ is zero.

By assumption $\alpha$ is nilpotent so we can choose $n \geqq 0$ with $\alpha^{p^{n}}=0$. Then Lemma 3.4 of [2] yields

$$
0=\alpha^{p^{n}}=\sum_{i=1}^{k} a_{i}^{p^{n}} g_{i}^{p^{n}}+\delta
$$

for some $\delta \in S$. If $a_{i} \neq 0$ then since the sum of the coefficients in the conjugacy class of $g_{i}^{p^{n}}$ must be zero in the above and since the 
contribution of $\delta$ to this sum is zero, we conclude that some $j \neq i$ must exist with $g_{j}^{p^{n}} \sim{ }_{G} g_{i}^{p^{n}}$.

LEMMA 2.3. Let $G$ be an L-linear group with $L G$ semisimple. Since $L$ is algebraically closed, $L G$ is a finite direct sum of full matrix rings over $L$ and we embed $L G$ in $L_{u}$ for some $u$ by placing the matrix rings of $L G$ in blocks along the diagonal of $L_{u}$. Then tr, the matrix trace map on $L_{u}$, yields a nondegenerate symmetric bilinear form $(\alpha, \beta)=\operatorname{tr} \alpha \beta$ on $L G$.

Proof. The form $(\alpha, \beta)=\operatorname{tr} \alpha \beta$ is certainly bilinear and symmetric. We need only show that it is nondegenerate on $L G$. Let $\alpha \in L G$ with $(\alpha, L G)=0$. Then

$$
\operatorname{tr}(L G) \alpha(L G)=\operatorname{tr} \alpha(L G)(L G)=\operatorname{tr} \alpha(L G)=0
$$

so every element of the ideal $(L G) \alpha(L G)$ has trace zero. But any nonzero ideal of $L G$ contains one of the full matrix ring and certainly all its elements cannot have trace 0 . Thus $\alpha$ must be zero and the lemma is proved.

We now obtain our generalization of Zalesskii's result by modifying the proof of [4]. It is apparent that the proof could be greatly simplified if we only knew that the radical was a nil ideal.

Lemma 2.4. Let $G$ be an L-linear group with $\boldsymbol{O}_{p}(G)=\langle 1\rangle$. Then $G$ has a normal subgroup $G_{0}$ of finite index and a representation of $G_{0}$ as an L-linear group in such a way that $L G_{0}$ is semisimple and if $\left[G_{0}: H\right]<\infty$ then $L G_{0}=L H$.

Proof. Since $\boldsymbol{O}_{p}(G)=\langle 1\rangle$, Lemma 1.1 (iii) implies that $G$ can be represented as an $L$-linear group with $L G$ semisimple. We now consider all normal subgroups $H$ of $G$ of finite index and all ways in which $H$ can be represented as an $L$-linear group with $L H$ semisimple and we choose $G_{0}$ to give the minimum possible dimension of $L G_{0}$.

Thus we have $G_{0} \triangleleft G,\left[G: G_{0}\right]<\infty$ and $G_{0}$ is an $L$-linear group with $L G_{0}$ semisimple. Furthermore, let $H$ be a subgroup of $G_{0}$ of finite index. Then $[G: H]<\infty$ so $H_{0}$, the intersection of the finitely many $G$-conjugates of $H$, is a normal subgroup of $G$ of finite index. Since $H_{0} \triangleleft G_{0}$ we have $L H_{0}$ semisimple by Lemma 1.1 (iv) and thus by the minimality of the dimension of $L G_{0}$ we have $L G_{0}=L H_{0}$ and hence $L G_{0}=L H$.

Proposition 2.5. Let $G$ be an L-linear group with $\boldsymbol{O}_{p}(G)=\langle 1\rangle$. Then $J K[G]$ is nilpotent. 
Proof. Let $G_{0}$ be the normal subgroup of $G$ of finite index given in the preceding lemma and let us write $L G_{0}$ as described in Lemma 2.3. Thus $L G_{0} \subseteq L_{u}$ and $\operatorname{tr}$ yields a nondegenerate bilinear form on $L G_{0}$. We show now that $K\left[G_{0}\right]$ is semisimple.

Suppose by way of contradiction that $\alpha=\sum_{i=1}^{k} a_{i} g_{i} \in J K\left[G_{0}\right]$ with $\alpha \neq 0$ and with the group elements $g_{i}$ distinct. If $x \in G_{0}$ then also $\alpha x=\sum_{i=1}^{k} a_{i} g_{i} x \in J K\left[G_{0}\right]$. Thus if $G_{1}$ is the finitely generated subgroup of $G_{0}$ given by $G_{1}=\left\langle g_{1}, g_{2}, \cdots, g_{k}, x\right\rangle$ then $\alpha x \in J K\left[G_{0}\right] \cap K\left[G_{1}\right] \subseteq J K\left[G_{1}\right]$ by Lemma 16.9 of [2]. We show now that for some $i \neq j, \operatorname{tr}\left(g_{i} x\right)=$ $\operatorname{tr}\left(g_{j} x\right)$.

Suppose this is not the case and let $G \widetilde{F}(p)$ denote the algebraic closure of $G F(p)$. Since $G_{1}$ is a finitely generated subgroup of $L_{u}$ we can find, by the Extension Theorem for Places, a place $\varphi: L \rightarrow$ $G \widetilde{F}(p) \cup\{\infty\}$ such that $\phi$ is finite on all the matrix entries of the generators of $G_{1}$ and their inverses and furthermore for all $i \neq j$, $\varphi\left(\operatorname{tr}\left(g_{i} x\right)\right) \neq \varphi\left(\operatorname{tr}\left(g_{j} x\right)\right)$. If $O$ denotes the corresponding valuation ring in $L$ then clearly $G_{1} \subseteq \mathcal{O}_{u}$ and $\varphi$ can be extended to a homomorphism $\varphi: \mathcal{O}_{u} \rightarrow G \widetilde{F}(p)_{u}$ and therefore $\varphi\left(G_{1}\right)$ is finite.

Consider the natural map $\eta: K\left[G_{1}\right] \rightarrow K\left[\varphi\left(G_{1}\right)\right]$. Since $\eta$ is an epimorphism, $\eta\left(J K\left[G_{1}\right]\right) \subseteq J K\left[\varphi\left(G_{1}\right)\right]$ and thus

$$
\eta(\alpha x)=\sum_{i=1}^{k} a_{i} \varphi\left(g_{i} x\right) \in J K\left[\varphi\left(G_{1}\right)\right]
$$

Now $\varphi\left(G_{1}\right)$ is finite so $J K\left[\varphi\left(G_{1}\right)\right]$ is nilpotent and therefore $\sum_{i=1}^{k} a_{i} \varphi\left(g_{i} x\right)$ is nilpotent. Thus Lemma 2.2 implies that for some $i \neq j$ and some integer $n, \varphi\left(g_{i} x\right)^{p^{n}} \sim{ }_{\varphi\left(G_{1}\right)} \varphi\left(g_{j} x\right)^{p^{n}}$. Let $\widetilde{\operatorname{tr}}$ denote the trace map in $G \tilde{F}(p)_{u}$. Since similar matrices have the same trace and since the fields have characteristic $p>0$ we conclude that

$$
\left[\tilde{\operatorname{tr}} \varphi\left(g_{i} x\right)\right]^{p^{n}}=\tilde{\operatorname{tr}}\left[\varphi\left(g_{i} x\right)^{p^{n}}\right]=\tilde{\operatorname{tr}}\left[\varphi\left(g_{j} x\right)^{p^{n}}\right]=\left[\tilde{\operatorname{tr}} \varphi\left(g_{j} x\right)\right]^{p^{n}}
$$

and thus $\tilde{\operatorname{tr}} \varphi\left(g_{i} x\right)=\tilde{\operatorname{tr}} \varphi\left(g_{j} x\right)$. But certainly $\tilde{\operatorname{tr}} \circ \varphi=\varphi \circ$ tr so we obtain

$$
\varphi\left(\operatorname{tr}\left(g_{i} x\right)\right)=\tilde{\operatorname{tr}} \varphi\left(g_{i} x\right)=\tilde{\operatorname{tr}} \varphi\left(g_{j} x\right)=\varphi\left(\operatorname{tr}\left(g_{j} x\right)\right)
$$

a contradiction.

We have, therefore, shown that for each $x \in G_{0}$ there exists some $i \neq j$ with $\operatorname{tr} g_{i} x=\operatorname{tr} g_{j} x$. For each $i \neq j$ let $T_{i j}$ be the $L$-subspace of $L G_{0}$ given by

$$
T_{i j}=\left\{\delta \in L G_{0} \mid \operatorname{tr}\left(g_{i}-g_{j}\right) \delta=0\right\} .
$$

Since $\operatorname{tr}$ yields a nondegenerate bilinear form we see that $T_{i j} \neq L G_{0}$ and by the above we have 


$$
G=\bigcup_{i \neq j} G \cap T_{i j} \cdot
$$

But then Lemma 2.1 with $S=\varnothing$ implies that $G_{0}$ has a subgroup $H$ of finite index with $L H \neq L G_{0}$, a contradiction. This shows that $K\left[G_{0}\right]$ is semisimple. Since $\left[G: G_{0}\right]<\infty$, Lemma 16.8 of [2] implies that $J K[G]$ is nilpotent and result follows.

3. A local situation. We now study a group $G$ with a rather special structure. We say $G$ has property $\left(^{*}\right)$ if $G$ has a normal series $G \supseteqq W \supseteqq P \supseteqq Z$ satisfying

1. $G / W$ is infinite cyclic.

2. $\bar{G}=G / P$ is an $L$-linear group with $\boldsymbol{O}_{p}(\bar{G})=\langle 1\rangle$.

3. $P$ is an abelian $p$-group.

4. $[P: Z]<\infty$ and $\mathrm{W}$ centralizes $Z$.

We say that $G$ has property (**) if $G$ satisfies all of the above and in addition

5. $P \cap \Delta(G)=\langle 1\rangle$.

Our aim is essentially to completely determine $J K[G]$ if $G$ satisfies $(*)$. We start by assuming that $G$ satisfies $(* *)$ and prove that $J K[G]$ is nilpotent. For the remainder of this section we assume that $G$ satisfies $\left({ }^{*}\right)$ and is given as above. We start by introducing some more notation.

Lemma 3.1. There exists a subgroup $G_{0}$ of $G$ of finite index with $G \supseteqq G_{0} \supseteqq P$ and such that

(i) $\bar{G}_{0}=G_{0} / P$ has a representation as an L-linear group with $L \bar{G}_{0}$ semisimple and with $L \bar{G}_{0}=L \bar{H}$ for all subgroups $\bar{H} \leqq \bar{G}_{0}$ of finite index.

(ii) $G_{0}$ centralizes the quotient $P / Z$.

(iii) If $W_{0}=G_{0} \cap W$ then $G_{0} / W_{0}$ is infinite cyclic.

Proof. The existence of a group $G_{0}$ satisfying (i) is an immediate consequence of Lemma 2.4. Furthermore, it is clear that this same property holds for any subgroup of $G_{0}$ of finite index which contains $P$. Now $G_{0}$ acts on finite group $P / Z$ and $P$ centralizes this quotient. Thus we may certainly replace $G_{0}$ by $C_{G_{0}}(P / Z)$ if necessary and then this new $G_{0}$ also satisfies (ii). Finally

$$
G_{0} / W_{0}=G_{0} /\left(W \cap G_{0}\right) \cong G_{0} W / W
$$

is a subgroup of finite index in the infinite cyclic group $G / W$ and the result follows.

We will show that $K\left[G_{0}\right]$ is semisimple. Thus by way of contradiction we assume now that $G_{0}$ is given as above and $J K\left[G_{0}\right] \neq 0$. 
LEMmA 3.2. There exists a nonzero element $\gamma=\alpha \beta \in J K\left[G_{0}\right] \cap$ $K\left[W_{0}\right]$ satisfying

(i) $\alpha=\hat{Q}$, the sum of all the elements of $Q$, where $Q$ is a finite subgroup of $P$. $W_{0}$.

(ii) $\beta=\sum_{i=1}^{n} a_{i} g_{i}$ where the $g_{i}$ are in distinct cosets of $P$ in

(iii) $\gamma$ centralizes $K[P]$.

Proof. By assumption $J K\left[G_{0}\right] \neq 0$ and since $G_{0} / W_{0}$ is infinite cyclic Theorem 17.7 of [2] implies that

$$
I=J K\left[G_{0}\right] \cap K\left[\mathrm{~W}_{0}\right]
$$

is a nonzero ideal of $K\left[W_{0}\right]$. Choose $\gamma \in I, \gamma \neq 0$ such that Supp $\gamma$ is contained in the smallest number $n$ of cosets of $P$. By multiplying $\gamma$ by a group element if necessary we may assume that one of these cosets is the identity coset. Thus

$$
\gamma=\sum_{i=1}^{n} \alpha_{i} g_{i}
$$

where $\alpha_{i} \in K[P]$ and $g_{1}=1, g_{2}, \cdots, g_{n}$ are in distinct cosets of $P$.

Let $Q$ be the subgroup of $P$ generated by the support of all the $\alpha_{i}$. Then $Q$ is a finitely generated and hence finite subgroup of the abelian $p$-group $P$. Therefore, as is well known, the unique minimal ideal of $K[Q]$ consists of all $K$-multiples of $\hat{Q}$ and thus $\hat{Q}$ is a multiple of $\alpha_{1}$ in $K[Q]$. By multiplying $\gamma$ on the left by this suitable factor we may clearly assume that $\alpha_{1}=\hat{Q}$. Let $h \in Q$. Then $(1-h) \alpha_{1}=0$ so $(1-h) \gamma \in I$ has support contained in a smaller number of cosets. This implies that $(1-h) \gamma=0$ for all $h \in Q$ and thus we have for all $i, \alpha_{i}=a_{i} \hat{Q}$ for some $a_{i} \in K$. This yields

$$
\gamma=\alpha \beta=\hat{Q}\left(\sum_{i=1}^{n} a_{i} g_{i}\right)
$$

and (i) and (ii) are proved.

Finally let $h \in P$. Since $P$ is abelian and $g_{1}=1$ we see $h^{-1} \gamma h-\gamma \in I$ has support in fewer cosets of $P$. By the minimality of $n$ we conclude that $h^{-1} \gamma h-\gamma=0$ for all $h \in P$ and (iii) follows.

We now define an even smaller subgroup of $G$. Again we fix the above notation for the remainder of this section. Let

$$
T=\left\{h \in Q \mid h \neq 1, C_{G_{0}}(h) \nsubseteq W_{0}\right\} \text {. }
$$

Now define the subgroup $G_{1}$ by 


$$
G_{1}=\bigcap_{h \leqslant T} W_{0} C_{G_{0}}(h)
$$

with the understanding that $G_{1}=G_{0}$ if $T=\varnothing$.

Lemma 3.3. Let $G_{1}$ be as above. Then

(i) $G_{0} \supseteqq G_{1} \supseteqq W_{0}$, $\left[G_{0}: G_{1}\right]<\infty$ and $G_{1} / W_{0}$ is infinite cyclic.

(ii) If $h \in T$ then $G_{1}=W_{0} C_{G_{1}}(h)$.

Proof. By definition we have $G_{0} \supseteqq G_{1} \supseteqq W_{0}$. Moreover, $G_{1} / W_{0}$ is the intersection of finitely many nonidentity subgroups of the infinite cyclic group $G_{0} / W_{0}$. Thus $G_{1} / W_{0}$ is infinite cyclic and $\left[G_{0}: G_{1}\right]<\infty$.

Finally let $h \in T$. Then $W_{0} \leqq G_{1} \subseteq W_{0} C_{G_{0}}(h)$ so

$$
G_{1}=W_{0}\left(G_{1} \cap \boldsymbol{C}_{G_{0}}(h)\right)=W_{0} \boldsymbol{C}_{G_{1}}(h)
$$

and the lemma is proved.

The reason for working with $G_{1}$ rather than $G_{0}$ will be apparent in the following result.

Lemma 3.4. Let $x \in G_{1}-W_{0}$ and let $\alpha$ be as above. Suppose that for infinitely many integers $s$ (positive or negative) there exists an integer $r=r(s) \geqq 1$ with

$$
\alpha \dot{\alpha}^{x-s} \alpha^{x^{-2 s}} \cdots \alpha^{x^{-r s}}=0 .
$$

Then for some $h \in T$ we have $x \in \boldsymbol{C}_{G_{1}}(h)$.

Proof. The assumption on $\alpha$ clearly implies that for each such $s$ the group $Q Q^{x^{-s}} Q^{x^{-2 s}} \cdots Q^{x^{-r s}}$ is not a direct product of the indicated factors. Since there are infinitely many such $s$ there are certainly infinitely many positive or infinitely many negative ones. Therefore, by Lemmas 3 and 4 of [1], there exists $h \in Q, h \neq 1$ and a positive integer $m$ with $x^{-m}$ or $x^{m}$ in $C_{G_{1}}(h)$ and hence $x^{m} \in \boldsymbol{C}_{G_{1}}(h)$. Now $x \in G_{1}-W_{0}$ and $G_{1} / W_{0}$ is infinite cyclic so $x^{m} \notin W_{0}$ and by definition of $T$ we must have $h \in T$.

Since $G_{1}=W_{0} C_{G_{1}}(h)$ by Lemma 3.3 (ii) we can write $x=w y$ with $w \in W_{0}$ and $y \in \boldsymbol{C}_{G_{1}}(h)$. Therefore, $y \in \boldsymbol{C}_{G_{1}}(h), x^{m}=(w y)^{m} \in \boldsymbol{C}_{G_{1}}(h)$ and since $W_{0}$ centralizes $P / Z$ we have $h^{w}=h z$ for some $z \in Z$. It then follows easily by induction on $i$ that

$$
h^{(w y)^{i}}=h z^{y} z^{y^{2}} \cdots z^{y^{i}}
$$

and therefore

$$
h=h^{(w y)^{m}}=h \boldsymbol{z}^{y} \boldsymbol{z}^{y^{2}} \cdots \boldsymbol{z}^{y^{m}}
$$


so we have $z^{y} z^{y^{2}} \cdots z^{y^{m}}=1$. We now conjugate this last expression by $y^{-1}$ and obtain

$$
z z^{y} \cdots z^{y^{m-1}}=1=z^{y} z^{y^{2}} \cdots z^{y^{m}} .
$$

Thus since $P$ is abelian we have $z=z^{y^{m}}$.

Since $G$ satisfies $\left(^{* *}\right)$ we know that $W$ centralizes $Z$ and thus we have $\boldsymbol{C}_{G_{1}}(z) \supseteqq\left\langle W_{0}, y^{m}\right\rangle$. Furthermore, $G_{1} / W_{0}$ is infinite cyclic and $y \notin W_{0}$ since $x \notin W_{0}$ so clearly $\left[G_{1}: C_{G_{1}}(z)\right]<\infty$. Hence $\left[G: C_{G}(z)\right]<\infty$ and we have $z \in P \cap \Delta(G)$. Again by assumption (**), $P \cap \Delta(G)=\langle 1\rangle$ so $z=1$. Finally $h^{x}=h^{w y}=h z^{y}=h$ so $x \in C_{G_{1}}(h)$ and the result follows.

Let denote the natural map $G_{0} \rightarrow \bar{G}_{0}=G_{0} / P$ and we extend this to the map $K\left[G_{0}\right] \rightarrow K\left[\bar{G}_{0}\right]$. Thus for $\beta=\sum_{i=1}^{n} a_{i} g_{i}$ as given before we have $\bar{\beta}=\sum_{i=1}^{n} a_{i} \bar{g}_{i}$. We now represent $\bar{G}_{0}$ as an $L$-linear group as in Lemma 3.1 (i) so that $L \bar{G}_{0}$ is semisimple.

LEMMA 3.5. We can embed $L \bar{G}_{0}$ in the matrix ring $L_{u}$ in such a way that tr, the matrix trace map on $L_{u}$, yields a nondegenerate symmetric bilinear form $L \bar{G}_{0}$. Futhermore, if for each $i \neq j$ we define $T_{i j}$ by

$$
T_{i j}=\left\{\bar{\delta} \in L \bar{G}_{0} \mid \operatorname{tr}\left(\bar{g}_{i}-\bar{g}_{j}\right) \bar{\delta}=0\right\}
$$

then $T_{i j}$ is a proper $L$-subspace of $L \bar{G}_{0}=L \bar{G}_{1}$.

Proof. The first part follows immediately from Lemma 3.1 (i) and Lemma 2.3. The second part about $T_{i j}$ follows from the nondegeneracy of the bilinear form and the fact that $\bar{g}_{i} \neq \bar{g}_{j}$ by Lemma 3.2 (ii). Finally $L \bar{G}_{0}=L \bar{G}_{1}$ by Lemma 3.1 (i).

Lemma 3.6. Let $x \in G_{1}-W_{0}$ and let $\beta$ be as above. Suppose that $\bar{\beta} \bar{x}^{s} \in K\left[\bar{G}_{1}\right]$ is nilpotent for all integers $s$ (positive or negative) with possibly finitely many exceptions. Then for some $i \neq j$ we have $\bar{x} \in T_{i j}$.

Proof. Since $x \in G_{1}-W_{0}$ and $\bar{G}_{1} / \bar{W}_{0}$ is infinite cyclic we see that $\langle\bar{x}\rangle$ is infinite. We consider $\langle\bar{x}\rangle$ as an $L$-linear subgroup of $\bar{G}_{0}$. Let $V$ denote the finite set of exceptional integers in the above and let

$$
S=\left\{\bar{x}^{v} \mid v \in V\right\} \text {. }
$$

Then $S$ is a finite subset of $\langle\bar{x}\rangle$ so clearly $[\langle\bar{x}\rangle: S]=\infty$. Now let $s$ be an integer not in $V$. Since

$$
\bar{\beta} \bar{x}^{s}=\sum_{i=1}^{n} a_{i} \bar{g}_{i} \bar{x}_{i}^{s}
$$


is nilpotent we conclude from Lemma 2.2 that for some $i \neq j$ and some integer $t \geqq 0$

$$
\left(\bar{g}_{i} \bar{x}^{s}\right)^{p^{t}} \sim{\overline{G_{1}}}_{1}\left(\bar{g}_{j} \bar{x}^{s}\right)^{p^{t}} .
$$

Thus since similar matrices have the same trace and since char $L=p>0$ we have

$$
\left(\operatorname{tr} \bar{g}_{i} \bar{x}^{s}\right)^{p^{t}}=\operatorname{tr}\left(\bar{g}_{i} \bar{x}^{s}\right)^{p^{t}}=\operatorname{tr}\left(\bar{g}_{j} \bar{x}^{s}\right)^{p^{t}}=\left(\operatorname{tr} \bar{g}_{j} \bar{x}^{s}\right)^{p^{t}} .
$$

Hence $\operatorname{tr} \bar{g}_{i} \bar{x}^{s}=\operatorname{tr} \bar{g}_{j} \bar{x}^{s}$ and $\bar{x}^{s} \in T_{i j}$.

We have therefore shown that

$$
\langle\bar{x}\rangle=S \cup \bigcup_{i \neq j}\left(\langle\bar{x}\rangle \cap T_{i j}\right)
$$

and since $[\langle\bar{x}\rangle: S]=\infty$, Lemma 2.1 implies that

$$
\langle\bar{x}\rangle=\bigcup_{i \neq j}\left(\langle\bar{x}\rangle \cap T_{i j}\right) \cdot
$$

This shows that $\bar{x} \in T_{i j}$ for some $i \neq j$ and the lemma is proved.

We now come to the main result of this section.

Proposition 3.7. Let $G$ be a group satisfying $\left(^{* *}\right)$. Then JK $[G]$ is nilpotent.

Proof. We use all the above notation and show first that $J K\left[G_{0}\right]=0$. If this is not the case then all of the above lemmas and notation apply.

Let $x \in G_{1}-W_{0}$ and let $s \neq 0$ be an integer (positive or negative). Since $G_{1} / W_{0}$ is infinite cyclic, the element $x^{-s}$ has infinite order modulo $W_{0}$. Since $\gamma \in J K\left[G_{0}\right] \cap K\left[W_{0}\right]$, Lemma 21.3 of [2] implies that for some integer $r=r(s) \geqq 1$ we have

$$
\gamma \gamma^{x^{-s}} \gamma^{x^{-2 s}} \cdots \gamma^{x^{-r s}}=0 \text {. }
$$

Now $\gamma=\alpha \beta$ so this yields

$$
\alpha \beta \alpha^{x^{-s}} \beta^{x^{-s}} \alpha^{x^{-2 s}} \cdots \alpha^{x^{-r s}} \beta^{x^{-r s}}=0 .
$$

By Lemma 3.2 (iii) $\gamma$ centralizes $K[P]$ and hence since $P \triangleleft G, \gamma^{x-i s}$ also centralizes $K[P]$.

We use this latter fact to rearrange the terms in the above product. First since the product is

$$
\gamma \gamma^{x^{-s}} \cdots \gamma^{x^{-\langle r-1) s}} \alpha^{x^{-r s}} \beta^{x-r s}
$$

we can shift the $\alpha^{x-r s}$ factor past all the $\gamma^{x^{-i s}}$ and obtain

$$
\alpha^{x^{-r s}} \gamma \gamma^{x^{-s}} \cdots \gamma^{x^{-\langle r-2\rangle s}} \alpha^{x^{-\langle r-1\rangle s}} \beta^{x^{-\langle r-1\rangle s}} \beta^{x^{-r s}} .
$$


We next shift the $\alpha^{x^{-\langle r-1) s}}$ term all the way to the left and continuing this process we clearly obtain

$$
\left(\alpha \alpha^{x^{-s}} \alpha^{x^{-2 s}} \cdots \alpha^{x^{-r s}}\right)\left(\beta \beta^{x^{-s}} \beta^{x^{-2 s}} \cdots \beta^{x^{-r s}}\right)=0 .
$$

Let $\sigma$ denote the above first factor and $\tau$ the second. Suppose that $\sigma \neq 0$. Now $P$ is an abelian $p$-group and char $K=p$ so $J K[P]$ is the unique maximal ideal of $K[P]$. This implies that every element of $K[P]-J K[P]$ is a unit in $K[P]$. If we now write $\tau$ as $\tau=\Sigma \tau_{i} y_{i}$ with $\tau_{i} \in K[P]$ and the $y_{i}$ in distinct cosets of $P, \sigma \tau=0$ and $\sigma \neq 0$ therefore implies that $\tau_{i} \in J K[P]$ and hence $\tau \in(J K[P]) K\left[G_{0}\right]$. But this ideal is precisely the kernel of the homomorphism $K\left[G_{0}\right] \rightarrow K\left[\bar{G}_{0}\right]$ and therefore $\bar{\tau}=0$. Thus

$$
0=\bar{\tau}=\bar{\beta} \bar{\beta}^{\bar{x}-s} \bar{\beta}^{\bar{x}-2 s} \ldots \bar{\beta}^{\bar{x}-r s}=\left(\bar{\beta} \bar{x}^{s}\right)^{r+1} \bar{x}^{-r s}
$$

and $\left(\bar{\beta} \bar{x}^{s}\right)^{r+1}=0$.

We have therefore shown that for each $s \neq 0$ either

$$
\alpha \alpha^{x^{-s}} \alpha^{x^{-2 s}} \cdots \alpha^{x^{-r s}}=0
$$

for some $r=r(s) \geqq 1$ or $\bar{\beta} \bar{x}^{s}$ is nilpotent. If the first fact occurs for infinitely many $s$ then by Lemma $3.4, x \in C_{G_{1}}(h)$ for some $h \in T$. If this first fact occurs for only finitely many $s$, then $\bar{\beta} \bar{x}^{s}$ is nilpotent for all but finitely many $s$ and Lemma 3.6 yields $\bar{x} \in T_{i j}$ for some $i \neq j$.

Observe that the above holds for any $x \in G_{1}-W_{0}$. Thus we see that

$$
\bar{G}_{1}=S \cup \bigcup_{i \neq j}\left(\bar{G}_{1} \cap T_{i j}\right)
$$

where

$$
S=\bar{W}_{0} \cup \bigcup_{h \in T} \overline{C_{G_{1}}(h)}
$$

We apply Lemma 2.1 and there are two possible conclusions. First there exists a subgroup $\bar{H}$ of $\bar{G}_{1}$ of finite index with $L \bar{H} \neq L \bar{G}_{1}$. But $\left[\bar{G}_{0}: \bar{G}_{1}\right]<\infty$ so $\left[\bar{G}_{0}: \bar{H}\right]<\infty$ and Lemma 3.1 (i) then yields $L \bar{G}_{1}=L \bar{G}_{0}=L \bar{H}$, and contradiction. Secondly we have $\left[\bar{G}_{1}: S\right]<\infty$ and this says that $\bar{G}_{1}$ is a finite union of cosets of the subgroups $\bar{W}_{0}$ and $\overline{\boldsymbol{C}_{G_{1}}(h)}$ for all $h \in T$. Then by Lemma 1.2 of [2] we see that one of these subgroups must have finite index in $\bar{G}_{1}$. Since $\bar{G}_{1} / \bar{W}_{0}$ is infinite cyclic we, therefore, have for some $h \in T,\left[\bar{G}_{1}: \overline{C_{G_{1}}(h)}\right]<\infty$. Moreover, [G: $\left.G_{1}\right]<\infty$ and $\boldsymbol{C}_{G_{1}}(h) \supseteqq P$ so this yields $\left[G: \boldsymbol{C}_{G_{1}}(h)\right]<\infty$. Thus $h \neq 1$ and $h \in P \cap \Delta(G)$, a contradiction since $G$ satisfies $\left(^{* *}\right)$.

We have therefore shown that $J K\left[G_{0}\right]=0$. Since $\left[G: G_{0}\right]<\infty$, 
Lemma 16.8 of [2] implies that $J K[G]$ is nilpotent and the proposition is proved.

4. The main theorem. In this section we prove our result. However, we first need a few additional facts about groups satisfying condition $(*)$.

Lemma 4.1. Let $G$ satisfy $\left(^{*}\right)$ and suppose that $P \cap \Delta(G)$ is finite. Then $J K[G]$ is nilpotent.

Proof. Let $Q=P \cap \Delta(G) \triangleleft G$ and consider $G / Q$. Then $G / Q$ has a normal series

$$
G / Q \supseteqq W / Q \supseteqq P / Q \supseteqq Z Q / Q
$$

and it is trivial to see that $G / Q$ has property (*). In addition $G / Q$ satisfies (**) as follows. Let $h \in P$ with $h Q / Q \in \Delta(G / Q)$. Then the $G$ conjugates of $h$ are contained in only finitely many cosets of $Q$. Since $Q$ is finite this implies that $h \in P \cap \Delta(G)=Q$ and $h Q / Q=1$. Thus $P / Q \cap \Delta(G / Q)=\langle 1\rangle$ and Proposition 3.7 implies that $J K[G / Q]$ is nilpotent.

Consider the natural map $K[G] \rightarrow K[G / Q]$. Since $Q$ is a finite $p$-group the kernel of this map is the nilpotent ideal $(J K[Q]) K[G]$. Moreover, we have

$$
J K[G] /(J K[Q]) K[G] \cong J K[G / Q]
$$

and since both $J K[G / Q]$ and $(J K[Q]) K[G]$ are nilpotent, the lemma is proved.

LEMMA 4.2. Let $Q$ be a periodic normal subgroup of a group $G$ with $Q \subseteq \Delta(G)$. Let $g, y \in G$ and suppose that $g Q / Q \in \Delta(G / Q)$. Then there exists an integer $m \geqq 1$ such that $y^{m}$ centralizes $g$.

Proof. Since $h Q / Q \in \Delta(G / Q)$ it follows that some power $y^{m^{\prime}}$ of $y$ with $m^{\prime} \geqq 1$ centralizes $g Q / Q$ and thus $\left(y^{m^{\prime}}, g\right) \in Q$. Moreover, since $Q$ is a periodic normal subgroup of $G$ contained in $\Delta(G)$, there exists a finite normal subgroup $H$ of $G$ with $\left(y^{m^{\prime}}, g\right) \in H$. This implies that $y^{m^{\prime}}$ normalizes the finite coset $H g$ and therefore some possibly bigger power $y^{m}$ of $y$ centralizes $g$.

At this point we could completely determine the structure of $J K[G]$ if $G$ satisfies $\left({ }^{*}\right)$. However, we will content ourselves with observing the following key fact. If $\alpha \in K[G]$ we let 
$p$-Supp $\alpha=\{h \in \operatorname{Supp} \alpha \mid h \neq 1$ has order a power of $p\}$.

Proposition 4.3. Let $G$ satisfy $\left(^{*}\right)$ and let $x \in G$. Suppose that $\alpha \in J K[G]$ with $1 \in \operatorname{Supp} \alpha$. Then there exists $h \in p$-Supp $\alpha$ and an integer $n \geqq 1$ such that $x^{n}$ centralizes $h$ and $h P / P \in \Delta^{p}(W / P)$.

Proof. Let $Q=P \cap \Delta(G) \triangleleft G$ and consider $G / Q$. Then $G / Q$ has a normal series

$$
G / Q \supseteqq W / Q \supseteqq P / Q \supseteqq Z Q / Q
$$

and it is trivial to see that $G / Q$ also satisfies (*). Suppose $z \in Z$ with $z Q / Q \in \Delta(G / Q)$ and choose $y \in G$ with $G=\langle W, y\rangle$. Then Lemma 4.2 applies and we conclude that $y^{m}$ centralizes $z$ for some $m \geqq 1$. Since $z \in Z$ we therefore have $C_{G}(z) \supseteqq\left\langle W, y^{m}\right\rangle$ and hence

$$
\left[G: C_{G}(z)\right]<\infty, z \in P \cap \Delta(G)=Q \text { and } z Q / Q=1 .
$$

We have shown that the group $G / Q$ satisfies $\left(^{*}\right)$ and in addition $Z Q / Q \cap \Delta(G / Q)=\langle 1\rangle$. Since $[P / Q: Z Q / Q]<\infty$ we therefore conclude that $P / Q \cap \Delta(G / Q)$ is finite and hence by Lemma 4.1,JK[G/Q] is nilpotent.

Write $\alpha$ as

$$
\alpha=\sum_{i=1}^{t} \alpha_{i} g_{i}
$$

with $\alpha_{i} \in K[Q]$ and with $g_{1}=1, g_{2}, \cdots, g_{t}$ in distinct cosets of $Q$ in $G$. Since $1 \in \operatorname{Supp} \alpha$ we can assume that $1 \in \operatorname{Supp} \alpha_{i}$ for all $i$ and hence $g_{i} \in \operatorname{Supp} \alpha$.

Suppose first that $\alpha_{1} \in J K[Q]$. Since $1 \in \operatorname{Supp} \alpha_{1}$ it follows that there exists $h \in \operatorname{Supp} \alpha_{1} \subseteq \operatorname{Supp} \alpha$ with $h \neq 1$. Then $h$ has order a power of $p$ and $h \in \Delta(G)$ so certainly $x^{n}$ centralizes $h$ for some $n$. Finally $h P / P=1 \in \Delta^{p}(W / P)$.

Now assume that $\alpha_{1} \notin J K[Q]$ and let $\sim$ denote the natural map $K[G] \rightarrow K[G / Q]$. Since $Q$ is an abelian $p$-group we see that the kernel of $\sim$ is $(J K[Q]) K[G]$ and therefore for each $i, \tilde{\alpha}_{i}=a_{i} \tilde{1}$ for some $a_{i} \in K$ and by assumption $a_{1} \neq 0$. Then

$$
\tilde{\alpha}=\sum_{i=1}^{t} a_{i} \widetilde{g}_{i} \in J K[G / Q]
$$

has $\widetilde{1}$ in its support. Furthermore, $J K[G / Q]$ is nilpotent so Theorem 20.2 (i) and Lemma 3.5 of [2] imply that for some $i \neq 1, \widetilde{g}_{i} \in \Delta^{p}(G / Q)$ and $\widetilde{g}_{i}$ has order a power of $p$. Since $Q$ is a $p$-group we see that $g_{i}$ has order a power of $p$ and by Lemma 4.2, $x^{n}$ centralizes $g_{i}$ for some 
$n \geqq 1$. Now $g_{i}$ has finite order a power of $p$ and $G / W$ is infinite cyclic so $g_{i} \in W$. Moreover, $g_{i} Q / Q$ has only finitely many conjugates in $G / Q$ so certainly $g_{i} P / P$ has only finitely many conjugates in $W / P$. Thus $g_{i} P / P \in \Delta^{p}(W / P)$ and the proposition is proved.

The following is well known.

Lemma 4.4. Let $G$ be a group and let $H$ be a normal $\Delta$-subgroup of $G$. Suppose that there exists an element $h \in H$ of order $p$ with $\left[G: C_{G}(h)\right]=l . f$. Then $J K[G] \cap K[H] \neq 0$.

Proof. Let $h$ and $H$ be given as above and let $H^{*}=\langle h\rangle^{H}$ be the normal closure of $\langle h\rangle$ in $H$. Then $H^{*}$ is a finite normal subgroup of $H$ whose order is divisible by $p$. We show that $J K\left[H^{*}\right] \subseteq J K[G]$. Since $J K\left[H^{*}\right] \neq 0$ and $J K\left[H^{*}\right] \subseteq K[H]$ this will yield the result.

Since $H^{*}$ is finite, it clearly suffices by Lemma 17.6 of [2] to show that if $S$ is a finitely generated subgroup of $G$ with $S \supseteqq H^{*}$ then $J K\left[H^{*}\right] \subseteq J K[S]$. Now by definition $\left[S: C_{S}(h)\right]<\infty$ so since $\boldsymbol{C}_{S}(h)$ clearly normalizes $H^{*}$ we have $\left[S: \boldsymbol{N}_{S}\left(H^{*}\right)\right]<\infty$. Let $N$ denote the core of $N_{S}\left(H^{*}\right)$ in $S$, that is the intersection of all conjugates of $N_{S}\left(H^{*}\right)$. Then [S: $\left.N\right]<\infty$ and $N \triangleleft S$. Since $H^{*} \subseteq S \cap H \triangleleft S$ and $S \cap H \subseteq N_{S}\left(H^{*}\right)$ we have $H^{*} \cong S \cap H \subseteq N$ and clearly $H^{*} \triangleleft N$. By Lemma 19.4 of [2], $J K\left[H^{*}\right] \subseteq J K[N]$ and by Theorem 16.6 of [2], $J K[N] \subseteq J K[S]$. Thus $J K\left[H^{*}\right] \leqq J K[S]$ and the result follows.

We can now prove our main theorem.

Proof of the Theorem. Let $G$ be an $L$-linear group. Suppose first that there exists an element $h \in \mathscr{L}(G)$ of order $p$ with $\left[G: C_{G}(h)\right]=$ l.f. Then by Lemmas 1.3 and 4.4 we have $J K[G] \cap K[\mathscr{L}(G)] \neq 0$ and hence $J K[G] \neq 0$.

Conversely let us assume that $J K[G] \neq 0$. There are three cases to consider.

Case 1. $\boldsymbol{O}_{p}(G)=\langle 1\rangle$.

By definition, $\mathscr{L}(G)=\Delta^{p}(G)$ here and by Proposition 2.3, JK $[G]$ is nilpotent. Thus by Theorem 20.2 there exists an element $h \in \Delta^{p}(G)$ of order $p$. Since $h \in \Delta^{p}(G)$ we have $\left[G: C_{G}(h)\right]<\infty$ and hence $\left[G: C_{G}(h)\right]=1 . f$.

Case 2. $G$ has a finite normal nonidentity $p$-subgroup.

Let this subgroup be $Q$. Then $Q \subseteq \boldsymbol{O}_{p}(G)$ so $Q \subseteq \Delta\left(\boldsymbol{O}_{p}(G)\right) \subseteq \mathscr{L}(G)$. Let $h$ be an element of order $p$ in $Q$. Then again $h \in \Delta(G)$ implies that $\left[G: C_{G}(h)\right]<\infty$ and hence $\left[G: C_{G}(h)\right]=$ l.f. 
Case 3. $P=O_{p}(G) \neq\langle 1\rangle$ and $G$ has no finite normal nonidentity $p$-subgroups.

Set $G^{*}=D_{G}(P)$. Since $J K[G] \neq 0$ and $P$ is nilpotent by Lemma 1.1 (i), it follows from results of [5], that $J K[G] \cap K\left[G^{*}\right] \neq 0$. Thus we may choose $\alpha \in J K[G] \cap K\left[G^{*}\right]$ with $1 \in \operatorname{Supp} \alpha$. We set $T=$ $p$-Supp $\alpha \cap \mathscr{L}(G)$.

Since $P$ is nilpotent and $P \neq\langle 1\rangle$ we have $\Delta(P) \neq\langle 1\rangle$ and hence by assumption $\Delta(P)$ is infinite. On the other hand, Lemma 1.2 (i) implies that $\left[\Delta(P):\left(\Delta(P) \cap Z\left(G^{*}\right)\right)\right]<\infty$. Thus we can choose $h_{0} \in$ $\Delta(P) \cap \boldsymbol{Z}\left(G^{*}\right)$ to be an element of order $p$. We show now that in the notation of [3]

$$
G=\sqrt{\boldsymbol{C}_{G}\left(h_{0}\right)} \cup \bigcup_{h \in T} \sqrt{\boldsymbol{C}_{G}(h)} .
$$

Let $x \in G$ and suppose first that $x G^{*} / G^{*}$ has infinite order. We consider the group $\widetilde{G}=\left\langle G^{*}, x\right\rangle$ and show that it satisfies condition $(*)$. First we have the normal series

$$
\widetilde{G} \supseteqq G^{*} \supseteq \Delta(P) \supseteqq Z
$$

where $Z=\Delta(P) \cap \boldsymbol{Z}\left(G^{*}\right)$. By assumption $\widetilde{G} / G^{*}$ is generated by $x G^{*} / G^{*}$ and is therefore infinite cyclic. This yields condition (1). Now $G^{*} \cap P=\Delta(P)$, and since $\widetilde{G} / G^{*}$ is infinite cyclic we have $\widetilde{G} \cap P=$ $G^{*} \cap P=\Delta(P)$. Thus since $G / P$ is an $L$-linear group by Lemma 1.1 (ii) so is $\widetilde{G} / \Delta(P) \cong \widetilde{G} P / P \subseteq G / P$. Again since $\widetilde{G} / G^{*}$ is infinite cyclic, $\boldsymbol{O}_{p}(\widetilde{G})=\boldsymbol{O}_{p}\left(G^{*}\right) \triangleleft G$ so $\boldsymbol{O}_{p}(\widetilde{G}) \leqq P \cap \widetilde{G}=\Delta(P)$ and therefore $\boldsymbol{O}_{p}(\widetilde{G} / \Delta(P))=$ $\langle 1\rangle$ so condition (2) is satisfied. Moreover, Lemma 1.2 (i) clearly yields (4). Finally $\Delta(P)$ has a center of finite index so by Lemma 2.1 of [2], $\Delta(P)^{\prime}$ is finite. Then this is a finite normal $p$-subgroup of $G$ so by assumption $\Delta(P)^{\prime}=\langle 1\rangle, \Delta(P)$ is abelian and condition (3) holds.

Thus $\widetilde{G}$ satisfies $(*)$. Now $\alpha \in J K[G] \cap K[\widetilde{G}] \subseteq J K[\widetilde{G}]$ by Lemma 16.9 of [2] so Proposition 4.3 implies that there exists $h \in p$-Supp $\alpha$ and an integer $n \geqq 1$ such that $x^{n}$ centralizes $h$ and $h \Delta(P) / \Delta(P) \in$ $\Delta^{p}\left(G^{*} / \Delta(P)\right)$. Note that the latter condition really says that $h \in \mathscr{P}(G)$. Thus $h \in T$ and

$$
x \in \bigcup_{h \in T} \sqrt{\boldsymbol{C}_{G}(h)} \cong \sqrt{\boldsymbol{C}_{G}\left(h_{0}\right)} \cup \bigcup_{h \in T} \sqrt{\boldsymbol{C}_{G}(h)} .
$$

Now let $x \in G$ with $x G^{*} / G^{*}$ of finite order. Then $x^{n} \in G^{*}$ for some $n \geqq 1$ and hence by the choice of $h_{0}, x^{n} \in C_{G}\left(h_{0}\right)$. Therefore, in this case also we have

$$
x \in \sqrt{\boldsymbol{C}_{G}\left(h_{0}\right)} \cong \sqrt{\boldsymbol{C}_{G}\left(h_{0}\right)} \cup \bigcup_{h \in T} \sqrt{\boldsymbol{C}_{G}(h)} .
$$

Thus we have show that 


$$
G=\sqrt{\boldsymbol{C}_{G}\left(h_{0}\right)} \cup \bigcup_{h \in T} \sqrt{\boldsymbol{C}_{G}(h)} .
$$

Therefore, since $G$ is a linear group, Proposition 7 of [3] implies that for some $g \in\left\{h_{0}\right\} \cup T$ we have $\left[G: C_{G}(g)\right]=$ l.f. Now by definition $\left\{h_{0}\right\} \cup T \subseteq \mathscr{L}(G)$ and hence $g \neq 1$ is an element of $\mathscr{L}(G)$ of order a power of $p$. Finally if $h$ is an element of order $p$ in $\langle g\rangle$, then $h \in \mathscr{L}(G)$ and $C_{G}(h) \supseteqq C_{G}(g)$ so $\left[G: C_{G}(h)\right]=$ l.f. and the theorem is proved.

5. Comments. The preceding proof is complicated by having to handle a number of small details. In each case if our knowledge of the situation was only a little more complete, a simplification of the proof would occur. For example, the unpleasantness of the place argument in Proposition 2.5 could be avoided if we knew that $J K[G]$ was a nil ideal. In addition much of the work in $\S 3$ would be simpler if we could assume that $P \subseteq \Delta(W)$ or in other words if we knew that for an $L$-linear group $G, \Delta(P) \subseteq \Delta\left(G^{*}\right)$ where $P=O_{p}(G)$ and $G^{*}=D_{G}(P)$.

Actually even a greater simplification would occur if only we could handle the equation

$$
G=\bigcup_{i=1}^{n} \sqrt{H_{i}} \cup \bigcup_{j=1}^{m}\left(G \cap T_{j}\right)
$$

where the $H_{i}$ are centralizer subgroups of $G$ and the $T_{j}$ are proper $L$-subspaces of $L G$ where $G$ is an $L$-linear group. We would of course want to conclude from the above that either $\left[G: H_{i}\right]=1$.f. for some $i$ or else that some subgroup of finite index has smaller linear span than $G$. However, this does not appear to be true at least in this generality. For example we have

EXAMPLE 5.1. Consider the $2 \times 2$ linear group over the complex numbers $C$ given by

$$
G=\left\{\left[\begin{array}{ll}
1 & 0 \\
a & b
\end{array}\right] \mid a, b \in C \text { and } b \text { is a root of unity }\right\} .
$$

Then $G$ has a normal subgroup $H$

$$
H=\left\{\left[\begin{array}{ll}
1 & 0 \\
a & 1
\end{array}\right] \mid a \in C\right\}
$$

isomorphic to $C^{+}$, the additive group of $C$. Note that $C^{+}$has no proper subgroups of finite index and thus if $\widetilde{G}$ is a subgroup of $G$ of finite index then $\widetilde{G} \supseteqq H$ and it follows easily that $C \widetilde{G}=C G$.

Let 


$$
T=\left\{\left[\begin{array}{ll}
d & 0 \\
a & d
\end{array}\right] \mid a, d \in C\right\} .
$$

Then $T$ is a proper $C$-subspace of $C G$ and $H \subseteq T$. Now suppose $x \in G-H$. Then $x=\left[\begin{array}{ll}1 & 0 \\ a & b\end{array}\right]$ for some $b \neq 1$ and thus clearly the matrix $x$ is similar to $\left[\begin{array}{ll}1 & 0 \\ 0 & b\end{array}\right]$. Since $b$ is a root of unity, this implies that $x$ has finite order and hence certainly $x \in \sqrt{\boldsymbol{C}_{G}(g)}$ where $g=\left[\begin{array}{rr}1 & 0 \\ 0 & -1\end{array}\right]$.

We have therefore shown that

$$
G=\sqrt{\boldsymbol{C}_{G}(g)} \cup(G \cap T)
$$

and certainly $\left[G: C_{G}(g)\right]$ is not locally finite since $C_{G}(g) \cap H=\langle 1\rangle$. Thus we see that we cannot conclude from such a decomposition of $G$ what we would like to.

Finally it would appear from the main result here and also the result for solvable groups given in [5] (or see [3] for a description of this fact) that $J K[G] \neq 0$ must imply in general that $G$ has a nonidentity normal 4 -subgroup. However, this is unfortunately not the case as we see below.

Let $p$ be a prime and let $A=Z_{p}$ be the cyclic group of order $p$ if $p>2$ and $A=Z_{4}$ if $p=2$.

LEMma 5.2. Let $H$ be an infinite p-group and let $G$ be the Wreath product $G=A$ ? $H$. If $N$ is a normal $\Delta$-subgroup of $G$ then $N$ is contained in the normal abelian subgroup of $G$ which in $\Sigma A$.

Proof. Write $G=W H$ where $W=\Sigma A$ is the direct sum of copies of $A$, one for each element of $H$. If $N \nsubseteq W$ choose $x \in N-W$ with $x^{p} \in W$. Then $N \supseteqq(x, W)$ but we see easily since $H$ is infinite that $\left[(x, W): \boldsymbol{C}_{(x, W)}(x)\right]=\infty$, a contradiction.

ExAmPle 5.3. Let $G_{1}$ be an infinite locally finite $p$-group and define $G_{1} \subseteq G_{2} \subseteq G_{3} \subseteq \cdots$ inductively by $G_{n+1}=A<G_{n}$. Then $G=$ $\mathrm{U}_{n=1}^{\infty} G_{n}$ is a locally finite $p$-group. If $N \neq\langle 1\rangle$ is a normal $\Delta$-subgroup of $G$ choose $n$ so that $N \cap G_{n} \neq\langle 1\rangle$. Then $N \cap G_{n+1}$ is a normal $\Delta$-subgroup of $G_{n+1}=A<G_{n}$ not contained in $\Sigma A$, a contradiction by the above lemma.

Thus $G$ has no nonidentity normal $\Delta$-subgroup. On the other hand, if $K$ is a field of characteristic $p$ then $J K[G]$ is the augmentation ideal of $K[G]$, since $G$ is a locally finite $p$-group. Therefore, $J K[G] \neq 0$. 


\section{REFERENCES}

1. D. S. Passman, On the semisimplicity of modular group algebras II, Canad. J. Math., 21 (1969), 1137-1145.

2. — Infinite Group Rings, Marcel Dekker, New York, 1971.

3. - On the semisimplicity of group rings of linear groups, Pacific J. Math., 47 (1973), 221-228.

4. A. E. Zalesskii, On group rings of linear groups, Siberian Math. J., 12 (1971), $246-250$.

5. - On the semisimplicity of a modular group algebra of a solvable group. Soviet Math., 14 (1973), 101-105.

Received May 16, 1972. Research supported in part by NSF contract GP-29432.

UNIVERSITY OF WISCONSIN, MADISON 


\section{PACIFIC JOURNAL OF MATHEMATICS}

\section{EDITORS}

RICHARD ARENS (Managing Editor)

University of California

Los Angeles, California 90024

R. A. Beaumont

University of Washington

Seattle, Washington 98105
J. DUGUNDJI*

Department of Mathematics

University of Southern California

Los Angeles, California 90007

D. Gilbarg and J. Milgram

Stanford University

Stanford, California 94305

\section{ASSOCIATE EDITORS}
E. F. BECKENBACH
B. H. NeumanN
F. WOLF
K. YosHIDA

\section{SUPPORTING INSTITUTIONS}

UNIVERSITY OF BRITISH COLUMBIA
CALIFORNIA INSTITUTE OF TECHNOLOGY
UNIVERSITY OF CALIFORNIA
MONTANA STATE UNIVERSITY
UNIVERSITY OF NEVADA
NEW MEXICO STATE UNIVERSITY
OREGON STATE UNIVERSITY
UNIVERSITY OF OREGON
OSAKA UNIVERSITY

UNIVERSITY OF BRITISH COLUMBIA

UNIVERSITY OF CALIFORNIA

MONTANA STATE UNIVERSITY

UNIVERSITY OF NEVADA

OREGON STATE UNIVERSITY

OSAKA UNIVERSITY
UNIVERSITY OF SOUTHERN CALIFORNIA

STANFORD UNIVERSITY

UNIVERSITY OF TOKYO

UNIVERSITY OF UTAH

WASHINGTON STATE UNIVERSITY

UNIVERSITY OF WASHINGTON

\section{AMERICAN MATHEMATICAL SOCIETY} NAVAL WEAPONS CENTER

* C. R. DePrima California Institute of Technology, Pasadena, CA 91109, will replace J. Dugundji until August 1974. 


\section{Pacific Journal of Mathematics}

\section{Vol. 48, No. $1 \quad$ March, 1973}

Jan Aarts and David John Lutzer, Pseudo-completeness and the product of Baire

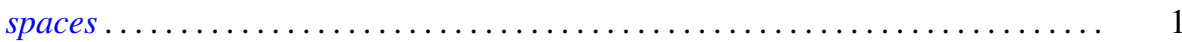

Gordon Owen Berg, Metric characterizations of Euclidean spaces ............ 11

Ajit Kaur Chilana, The space of bounded sequences with the mixed topology ..... . 29

Philip Throop Church and James Timourian, Differentiable open maps of

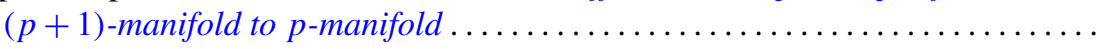

P. D. T. A. Elliott, On additive functions whose limiting distributions possess a finite

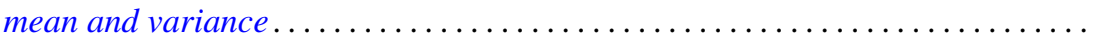

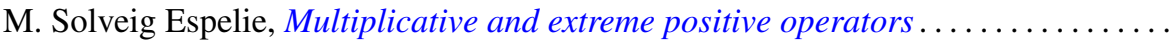

Jacques A. Ferland, Domains of negativity and application to generalized convexity

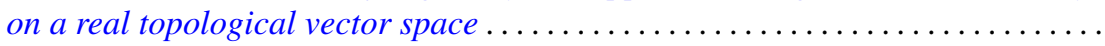

Michael Benton Freeman and Reese Harvey, A compact set that is locally holomorphically convex but not holomorphically convex ...............

Roe William Goodman, Positive-definite distributions and intertwining

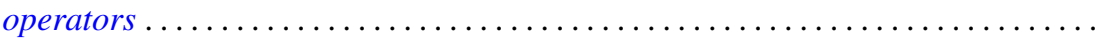

Elliot Charles Gootman, The type of some $C^{*}$ and $W^{*}$-algebras associated with

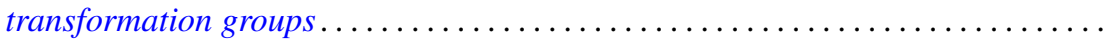

David Charles Haddad, Angular limits of locally finitely valent holomorphic

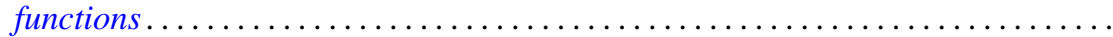

William Buhmann Johnson, On quasi-complements .

William M. Kantor, On 2-transitive collineation groups of finite projective spaces...

Joachim Lambek and Gerhard O. Michler, Completions and classical localizations of right Noetherian rings

Kenneth Lamar Lange, Borel sets of probability measures ......

David Lowell Lovelady, Product integrals for an ordinary differential equation in a Banach space

Jorge Martinez, A hom-functor for lattice-ordered groups .........

W. K. Mason, Weakly almost periodic homeomorphisms of the two sphere ....

Anthony G. Mucci, Limits for martingale-like sequences .......

Eugene Michael Norris, Relationally induced semigroups ...

Arthur E. Olson, A comparison of c-density and $k$-density ......

Donald Steven Passman, On the semisimplicity of group rings of linear groups.

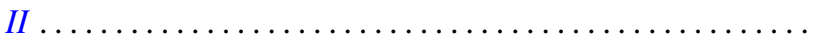

Charles Radin, Ergodicity in von Neumann algebras .

P. Rosenthal, On the singularities of the function generated by the Bergman operator of the second kind.

Arthur Argyle Sagle and J. R. Schumi, Multiplications on homogeneous spaces,

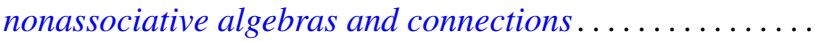

Leo Sario and Cecilia Wang, Existence of Dirichlet finite biharmonic functions on

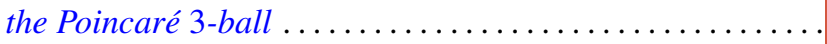

Ramachandran Subramanian, On a generalization of martingales due to Blake ..

Bui An Ton, On strongly nonlinear elliptic variational inequalities.

Seth Warner, A topological characterization of complete, discretely valued

fields. 